\title{
Autoformación de las ONG en transparencia y rendición de cuentas en Ecuador
}

\author{
Susan Appe \\ Binghamton University (Nueva York, Estados Unidos) \\ sappe@binghamton.edu \\ Daniel Barragán \\ Universidad de Los Hemisferios (Quito, Ecuador) \\ daniel.barragan@udeloshemisferios.com
}

Fecha de presentación: 28 de abril de 2016 • Fecha de aceptación: 22 de julio de 2017

Artículo de investigación 


\section{Susan Appe y Daniel Barragán}

\section{Resumen}

El artículo se enfoca en la inexistencia de programas de EGONG en asuntos relacionados con la transparencia y la rendición de cuentas en el caso de Ecuador, y cómo la demanda existente está siendo abordada por las propias organizaciones pero no está siendo visualizada por el sector universitario. Se identifican tres hallazgos principales: en primer lugar, la generación de alianzas fue clave para hacer frente a la oferta limitada de programas formales de EGONG; el potencial de réplica de los conocimientos fue uno de los objetivos de los procesos estudiados; y la evaluación de los procesos de los programas fue fundamental para dinamizar nuevos programas.

Palabras clave: Programas educativos, gestión, ONG, transparencia y rendición de cuentas, Ecuador.

JEL: L31 Instituciones sin fines de lucro, organismos no gubernamentales (ONG); M14 Ccultura corporativa; responsabilidad social corporativa.

\section{Abstract}

The article focuses on the lack of EGONG programs in matters related to transparency and accountability in the case of Ecuador, and how the existing demand is being addressed by the organizations themselves but it is not being visualized by the university sector. Three principal discoveries are identified: first of all, generating alliances was essential to addressing the limited supply of formal EGONG programs; the potential for the replication of knowledge was one of the objectives on the studied process, and the evaluation of the processes of the programs was fundamental to energize new programs.

Keywords: Educational programs, management, NGO, transparency and accountability, Ecuador.

JEL: L31 nonprofit institutions, NGOs, Social Entrepreneurship; M14 Corporate culture; corporate social responsibility.

\section{Resumo}

$\mathrm{O}$ artigo se foca na inexistência de programas EGONG em assuntos relacionados à transparência e à prestação de contas no Equador, focando-se também na forma como a demanda existente está sendo abordada pelas próprias organizações, mas não está sendo visualizada pelo setor universitário. Foram identificadas três principais descobertas: em primeiro lugar, a geração de alianças foi essencial para enfrentar a oferta limitada de programas formais de EGONG. Além disso, o potencial de réplica dos conhecimentos foi um dos objetivos dos processos estudados, e a avaliação dos processos dos programas foi fundamental para dinamizar novos programas.

Palavras-chave: Programas educativos, gestão, $\mathrm{ONG}$, transparência e prestação de contas, Equador.

JEL: L31 Instituições sem fins lucrativos, organizações não-governamentais (ONGs); M14 A cultura corporativa; responsabilidade social corporativa. 


\section{Introducción}

n América Latina existe una demanda de programas educativos en gestión de organizaciones no gubernamentales (ONG), sin fines de lucro, que les permita responder oportuna y efectivamente a las demandas sociales, a los requerimientos de las agencias donantes y al incremento de regulaciones gubernamentales hacia las ONG en la región con el fin de implementar prácticas de gestión transparentes y mecanismos de rendiciones de cuentas; pero también como resultado de procesos de reflexión interna de las propias organizaciones que están interesadas en internalizar valores como la ética, la transparencia, y la rendición de cuentas en sus operaciones, no solo como un compromiso por autorregularse sino también para mostrar coherencia con su accionar (Cruz y Pousadela 2008).

De igual forma las ONG en esta región han sido reconocidas en las últimas décadas por jugar un papel clave en la provisión de bienes y servicios públicos (Brautigam y Segarra 2007; Arcos y Palomeque 1997; Heinrich 2007; World Bank 2005; Barragán 2010; Chiriboga 2014; Belletini 2015); sin embargo, a pesar del importante rol de las organizaciones en la sociedad, y de los bienes y servicios que proporcionan, hay una deficiencia en la oferta de programas formales de educación en gestión de ONG (EGONG) en las instituciones de la educación superior en la región (Mirabella y otros 2007).

El presente artículo profundiza un trabajo previo publicado (Appe y Barragán 2013) en el que se reconoce la limitada oferta de programas formales de EGONG en América Latina, pero también observa que desde el estudio comparativo de Mirabella y otros (2007) varios programas de EGONG -dentro y fuera de instituciones de la educación superior- han emergido en la región. El artículo se enfoca, y profundiza, en las limitaciones de los programas de EGONG en Ecuador en asuntos relacionados con la transparencia y la rendición de cuentas. Rubaii y Pliscoff (2013) enfatizaron que, en el contexto de 
América Latina, la profesionalización en la prestación de bienes y servicios públicos abarca no solo el conocimiento y las habilidades necesarias para una buena gobernanza, sino también valores de servicio público como la rendición de cuentas y la transparencia, entre otros; en este contexto, las ONG, por presiones sociales, también deben considerar los valores del servicio público.

El artículo presenta una revisión de la literatura sobre EGONG, transparencia y rendición de cuentas; continúa con el tratamiento metodológico para luego exponer las limitaciones de los programas de EGONG en Ecuador. Identifica cuatros casos con los que se busca franquear la brecha existente en la oferta de EGONG para los temas de transparencia y rendición de cuentas. Finalmente, se presentan las conclusiones sobre lecciones aprendidas que pueden contribuir al desarrollo de programas y estrategias de EGONG en Ecuador y en América Latina.

\section{Revisión de literatura}

En el transcurso de las últimas dos décadas, la literatura sobre educación en administración pública está dando más atención a programas de EGONG y sus contenidos curriculares (Burlingame 2009; Mirabella y Young 2012; O’Neill 2005; Wish y Mirabella 1998). Así, si bien hay un incremento en los enfoques de la literatura comparativa que investiga EGONG (Donmoyer y otros 2012; Jackson 2009; Mirabella 2007; Mirabella y otros 2007; Schmitz, Raggo y Bruno-van Vijfeijken 2011), esta todavía está enfocada en el contexto de los Estados Unidos y en el crecimiento de los programas de EGONG (O’Neill 2005) a base de las expectativas profesionales en organizaciones sin ánimo de lucro (Bies y Blackwood 2007; O’Neill 2005). Programas de este tipo cada vez son relevantes debido a las mayores exigencias de profesionalizar el trabajo en el tercer sector. El hecho de que las ONG se hayan expandido a nivel global, implica que este crecimiento también presione a la oferta de programas de formación para directivos y líderes de ONG. Las habilidades requeridas por los dirigentes incluyen la capacidad de recaudación de fondos, la planificación estratégica y contabilidad, entre otros (Mirabella y otros 2007), y, dependiendo del contexto de un país, puede implicar conocimientos o herramientas particulares. 
Adicionalmente a la literatura de educación sobre administración pública y de EGONG en particular, existen investigaciones sobre transparencia, rendición de cuentas, y autorregulación de ONG que son relevantes a nivel Iberoamericano. Estos estudios contribuyen, para América Latina, en la discusión académica emergente relacionada con los mecanismos de rendiciones de cuentas y autorregulación de organizaciones sin ánimo de lucro a nivel global (Bies 2010; Bothwell 2001; Ebrahim 2003; Prakash y Gugerty 2010). Estudios recientes han descrito y explicado la autorregulación en los Estados Unidos (Bothwell 2001), Asia (Sidel 2005), África (Gugerty 2008), y Europa (Bies 2010); sin embargo, pocas investigaciones académicas en administración pública han cubierto lass tendencias en Iberoamérica. Más bien, varios institutos de investigación en América Latina han abierto la puerta a investigaciones que abordan la profesionalización de las ONG como uno de los retos del contexto actual. El Instituto de Innovación Social de ESADE en España y el Centro de Innovación Social de la Universidad de San Andrés en Argentina han revelado evidencia de la profesionalización y necesidad de fortalecimiento de su gestión. Otros, como el Instituto de Comunicación y Desarrollo en Uruguay o el Instituto Tecnológico Autónomo de México, han realizado contribuciones importantes en el desarrollo de currículos e investigaciones aplicadas.

En el caso de Ecuador existe limitada literatura sobre el tema. En 2011, Arias realizó una investigación que analiza la situación de la transparencia y rendición de cuentas en las ONG de Ecuador, aportando algunos elementos importantes. A nivel conceptual propone definiciones aterrizadas al contexto de Ecuador. A la rendición de cuentas, por ejemplo, se la concibe como "la manera oportuna cómo las ONG explican y se responsabilizan por la gestión, resultados, impactos y consecuencias de sus acciones ante las distintas audiencias y actores involucrados" (Arias 2011, 36). Esta aproximación, básicamente orientada en el dar cuenta de lo actuado por una organización, contrasta con enfoques de mucho mayor alcance en los que se abordan consideraciones como la calidad de las relaciones, el poder e influencia en la vida de terceros o la necesidad de contar con mecanismos que generen contrapesos a ese poder (Zadek 2006; Bonbright 2007). Estas diferencias, desde lo conceptual, evidencian otro resultado de la investigación: el poco conocimiento y capacidad técnica específica relacionada con la práctica de la transparencia y la rendición de cuentas, así como la necesidad de formar 
y fortalecer capacidades - no solo a nivel de prácticas sino también a nivel conceptual- en las organizaciones.

Con este antecedente, el artículo se centra en el caso de Ecuador y se fundamenta en una investigación previa sobre el contexto y el ambiente habilitante de Ecuador para la generación de oportunidades de formación para las ONG y sus gestores. Se identificaron tres medios importantes a través de los cuales las ONG responden a la percepción de que hay una falta de oportunidades de formación. En primer lugar, la conformación de una instancia colectiva y la creación de espacios para que las ONG mantengan discusiones y acciones a nivel de sector. En segundo lugar, Appe y Barragán (2013) identificaron un interés emergente para la producción de conocimiento sobre el sector por las propias ONG - esto incluye documentos de posición sobre cuestiones de política, investigaciones sobre el sector e informes de rendición colectiva de cuentas-. En tercer lugar, la existencia de cursos impulsados por las propias organizaciones (Appe y Barragán 2013) presentan ejemplos de las oportunidades de formación promovidas por las propias ONG y que se detallan en el presente documento.

Como se ha mencionado, las estrategias de las ONG se dieron en respuesta a factores coyunturales, pero también a la percepción de que no existen oportunidades formales de EGONG para los gestores de las organizaciones en Ecuador. Esto fue explorado con mayor profundidad por Appe (2015) mediante un análisis sistemático de contenidos para una más sólida comprensión de los planes de estudio de EGONG en el ámbito universitario en Ecuador. De forma puntual, Appe (2015) examinó el único curso en modalidad abierta sobre EGONG ofrecido por el Área Académica de Gestión de la Universidad Andina Simón Bolívar, Sede Ecuador (UASB). El trabajo fue construido sobre los estudios de Young y Grinsfelder (2011) y Mirabella y Young (2012) sobre la relación entre la ubicación y el contenido de los programas de EGONG. Young y Grinsfelder (2011) examinaron la literatura sobre negocios, sector público y emprendimiento social para encontrar las coincidencias y diferencias en las habilidades requeridas y determinaron tres grupos de habilidades: de mercado, políticas y de gestión. Posteriormente, Mirabella y Young (2012) ampliaron la clasificación propuesta por Young y Grinsfelder para habilidades de gestión e incluyeronlas habilidades filantrópicas, de gestiones genéricas y de liderazgo. Las habilidades de mercado permiten a los gestores tener éxito 
en un entorno de mercado; las políticas son para la negociación en entornos para la provisión de bienes y servicios públicos; las habilidades filantrópicas son "requeridas para la adquisición de recursos filantrópicos" o "la gestión de voluntarios" (Mirabella y Young 2012, 48) y las habilidades de gestión genéricas para el funcionamiento de una organización. Además, se necesitan habilidades de liderazgo para lograr el liderazgo fuera de la dirección de la organización interna. Sobre la base de este marco, además, encontraron que la ubicación de un programa de emprendimiento social -en una escuela de negocios versus uno de asuntos públicos- influye en el contenido curricular del programa. Por lo tanto, la ubicación de los programas es una variable importante para la definición de los contenidos de los programas de EGONG.

Appe (2015) concuerda con los hallazgos de Appe y Barragán (2013) en cuanto a las limitaciones de la oferta de EGONG en el ámbito universitario en Ecuador. La autora concluye que la EGONG en el sistema universitario no está cumpliendo plenamente la demanda existente en Ecuador por lo que realizó varias recomendaciones para el sistema universitario de Ecuador con relación a la EGONG. En primer lugar, afirma que cursos como el ofertado por la UASB necesitan recoger retroalimentación de los participantes y llevar a cabo evaluaciones con el fin de dar respuesta a los requerimientos de actualidad y necesidades de las organizaciones en contextos específicos. En segundo lugar, recomienda la reestructuración de las áreas temáticas cubiertas por los cursos para incluir temas relacionados con la ética, la rendición de cuentas y la transparencia. En tercer lugar, sugiere una expansión del curso en la UASB, tanto en su duración como la inclusión de contenidos en otros módulos teóricos y la utilización de casos y para fortalecer la calidad del programa.

De hecho, un reciente estudio empírico sobre educación de posgrado en asuntos públicos indica que, entre las especializaciones ofertadas en Estados Unidos, la más común es en gestión de entidades sin ánimo de lucro (58\% de los programas); sin embargo, el mismo estudio concluye que no existen programas para organizaciones no lucrativas en una muestra de 26 universidades en América Latina (Sanabria, Rubaii y Purón 2016).

En este marco, se busca contribuir a la investigación relacionada con el contexto para la promoción de oportunidades de EGONG en América Latina y específicamente en Ecuador y se profundiza el análisis, en oferta y contenidos, sobre cursos desarrollados por las propias ONG en Ecuador. 


\section{Metodología}

Ecuador es un caso particularmente interesante porque los programas formales de EGONG no están en la oferta académica de instituciones de educación superior y existe un proceso de reforma continua de la regulación a las organizaciones de la sociedad civil, promovido por el gobierno, con énfasis en promover un mayor control, transparencia y rendiciones de cuentas (Barragán 2010; 2011). En este contexto, el artículo se enfoca en programas de EGONG en Ecuador que abordan la transparencia y rendiciones de cuentas, para lo cual se han identificado varias estrategias implementadas por líderes de ONG para generar capacidades frente a las demandas del contexto.

La información proviene de varios cursos en Ecuador organizados por el Centro Ecuatoriano de Derecho Ambiental (CEDA) con el apoyo del Instituto de Comunicación y Desarrollo (ICD), de la Facultad Latinoamericana de Ciencias Sociales (FLACSO) y de la Universidad Casa Grande; así como otro realizado por Grupo Faro con apoyo de la Universidad Técnica Particular de Loja (UTPL) y la Fundação Getúlio Vargas de Brasil, así como de entrevistas con los gestores de los cursos, archivos institucionales y las páginas institucionales de los centros, que permiten presentar una descripción del caso de Ecuador a fin de buscar nuevas oportunidades de investigación y generar recomendaciones aplicables a Ecuador.

\section{Experiencias de educación en gestión de las ONG sobre transparencia y rendición de cuentas}

Si bien el fortalecimiento de capacidades está en el centro de las preocupaciones de las organizaciones sin fines de lucro (Ott y Dicke 2012), en el actual -y cambiante- entorno existe una considerable preocupación de que el tercer sector pueda carecer de la capacidad y experiencia técnica para mantenerse al día con los retos que el entorno exige (De Vita, Fleming y Twombly 2012).

En Ecuador, el desarrollo de capacidades es también uno de los temas más relevantes para las organizaciones, principalmente por la falta de oportunidades de formación y entrenamiento para sus propios gestores. Entendiéndose este fortalecimiento como el de "incrementar la habilidad de una 
organización para lograr su misión” (Wing 2004, 155), en el país es considerado como un proceso para construir conocimiento y competencias relacionados con la gestión organizacional. En particular, una de las principales debilidades de la sociedad civil en Ecuador es el poco conocimiento y capacidad técnica especifica relacionada a la práctica de la transparencia y la rendición de cuentas (Arias 2011). Esta tendencia, en menor medida, también es compartida en otros países de Iberoamérica como Argentina y España (Carreras y Sureda 2011; Indij y Lomé 2013).

Las propias organizaciones han visto la necesidad de planificar y ejecutar procesos de capacitación que aborden estos temas, desde un enfoque práctico pero también a medida de los retos que el contexto sociopolítico les exige. Esto lo refuerzan De Vita, Fleming y Twombly (2012) al señalar que, debido a las necesidades de la organización y las variantes condiciones del entorno, los enfoques para la creación de capacidades deben ser personalizados y flexibles. Otra motivación es la capacidad de liderar la promoción y trabajo en los temas de transparencia y rendición de cuentas hacia sus grupos beneficiarios o en el marco de procesos colectivos.

Para el presente artículo, se consideraron cuatro cursos sobre transparencia y rendición de cuentas en orden cronológico, los cuales se sistematizan en la tabla 1.

\section{0: conceptos y mecanismos para la transparencia y rendición de cuentas}

Este curso virtual fue promovido por una organización de sociedad civil con base en Quito en alianza con el ICD. El curso abordó, desde una visión introductoria, el marco teórico de la transparencia, algunas experiencias en América Latina y buenas prácticas de transparencia y rendición de cuentas.

A nivel metodológico, el curso estaba estructurado en siete módulos, con una duración de tres meses e incluyó charlas, bibliografía obligatoria y recomendada, y ejercicios enfocados en la reflexión y evaluación de las experiencias propias de los participantes.

La convocatoria del curso generó alta expectativa y permitió seleccionar 42 participantes provenientes de distintas ciudades y zonas del país, así como 
de distinto tipo de organizaciones (fundaciones, $\mathrm{ONG}$, colegios profesionales y ONG internacionales), que ofrecían una alta posibilidad de aplicación real y práctica de los conocimientos y de las herramientas aprendidas en las operaciones de sus propias organizaciones, lo cual quedó demostrado en la evaluación y medición de efectos; también se evidenció la necesidad de las organizaciones para fortalecer sus capacidades en herramientas de gestión y en transparencia y rendición de cuentas.

\section{1: herramientas para la transparencia y rendiciones de cuentas}

Este curso teórico-práctico se realizó, en alianza, entre una organización de sociedad civil y la FLACSO; se orientó a generar mayores capacidades, herramientas y recursos a partir del debate y el intercambio de conocimientos, a fin de avanzar en los aspectos de transparencia y rendición de cuentas de las organizaciones de la sociedad civil (OCS). El énfasis era profundizar en herramientas que puedan desarrollar habilidades en las organizaciones.

El curso se desarrolló de forma semipresencial en aproximadamente dos meses y medio, con una duración de 68 horas-clase en aula, las cuales se complementaron con foros presenciales y virtuales con la participación de 25 representantes de organizaciones de varias zonas del país. La parte presencial tuvo seis módulos con temas y herramientas clave para fortalecer la legitimidad, la transparencia y la rendición de cuentas de las organizaciones (política de transparencia; marco legal; herramientas para el monitoreo y evaluación de impacto; modelo de gestión en responsabilidad social; comunicación para la transparencia y diálogo intersectorial), y que a su vez permitieron profundizar conocimientos y habilidades.

El curso incluyó una metodología similar al descrito previamente. A pesar de que se realizó de forma semi-presencial, incluyó también un formato de charlas, bibliografía requerida y recomendada; así como una evaluación de los docentes como parte del proceso formativo requerido por FLACSO para la emisión de certificados. Como el primer curso, la evaluación del curso realizada por los participantes demostró que hubo experiencias positivas de los participantes. 


\section{4-2015: responsabilidad social como un modelo de gestión que fomenta y facilita la transparencia y rendición de cuentas}

Este curso sumó esfuerzos entre una OCS, una red de empresas y organizaciones por la responsabilidad social, una red local de organizaciones y la Universidad Casa Grande de Guayaquil, para ofrecer un proceso de aprendizaje y fortalecimiento en responsabilidad social y generación de alianzas estratégicas orientadas a la creación de capacidades y prácticas institucionales.

Con una modalidad presencial se abordaron tres grandes módulos orientados al fortalecimiento de la capacidad de gestión, sostenibilidad y legitimidad de organizaciones: a) modelo de gestión en responsabilidad social, b) reporte de sostenibilidad como herramienta de rendición de cuentas, y, c) comunicación estratégica para la construcción de diálogo, generación de alianzas y legitimidad social de las OSC.

El proceso de aprendizaje promovió -en 23 participantes- la responsabilidad social como un modelo de gestión que fomenta y facilita la transparencia y rendición de cuentas como un medio para construir la propia legitimidad de las organizaciones y abonar a su sostenibilidad en un contexto de cambio. Si las organizaciones están conscientes de su impacto en los ámbitos económico, social y ambiental se les facilitará la gestión de sus impactos y potenciar su rol social.

\section{5: innovación y transparencia en organizaciones de la sociedad civil}

La iniciativa Innovacción, ${ }^{1}$ impulsada por una OCS, promovió un curso semipresencial sobre "Innovación y transparencia en organizaciones de la sociedad civil (OSC)" y otro similar para los gobiernos autónomos descentralizados (GAD), específicamente municipios, para lo cual se generaron

1. Mayor información de la iniciativa en «ttp://innovaccion.ec/〉. 
alianzas con dos centros académicos: la Universidad Técnica Particular de Loja (UTPL) de Ecuador y la Fundação Getúlio Vargas de Brasil.

La coordinadora de Innovacción, Cecilia Lincango (2016), en comunicación personal señaló que este programa se concibió como un proceso de generación del conocimiento para lograr cambios, a través de la articulación de los tres sectores sociales -Estado, empresa y sociedad-en una lógica de corresponsabilidad y trabajo conjuntos para alcanzar un verdadero desarrollo y la construcción de una sociedad democrática.

El programa contó con la participación de 35 personas y estuvo dirigido para organizaciones; se estructuró para fortalecerlas en un momento y escenario político complejo: regulación restrictiva, cierre de organizaciones por la imposición de cambio de disposiciones en sus estatutos, salida de la cooperación internacional, entre otras situaciones. Los temas abordados incluyeron: a) transversalización del enfoque de género en la política pública; b) participación ciudadana; c) transparencia y rendición de cuentas; y d) gestión de proyectos (entendido como la posibilidad de generar un proyecto en función de un objetivo más grande, y no solo como fuente recursos para subsistir sino para aportar a un marco de desarrollo local más amplio).

Un módulo completo abordó la temática de transparencia y rendición de cuentas, tanto desde el marco normativo como su implementación práctica desde una lógica de herramienta de evaluación y gestión de las organizaciones.

Este programa fue replicado en 2016 con base en los aprendizajes desarrollados en su primera edición, con un total de 44 participantes.

\section{Discusión}

Este artículo presenta tres hallazgos concretos frente a la oferta limitada de programas formales de EGONG en América Latina, con énfasis en el caso de Ecuador. De las iniciativas analizadas, se encontró que los nuevos programas de EGONG demuestran la importancia de la construcción de alianzas entre distintos actores y el potencial de réplica como objetivo común en todos los cursos analizados.

Hallazgo No. 1: Las cuatro experiencias analizadas fueron promovidas por OCS e impulsaron alianzas entre organizaciones de sociedad civil y cen- 
Tabla 1

Casos analizados

\begin{tabular}{|c|c|c|c|c|}
\hline Año & Curso & Temáticas que aborda & Modalidad & $\begin{array}{l}\text { Número de } \\
\text { participantes }\end{array}$ \\
\hline 2010 & $\begin{array}{l}\text { Conceptos y } \\
\text { mecanismos } \\
\text { para la } \\
\text { transparencia y } \\
\text { rendición } \\
\text { de cuentas. }\end{array}$ & $\begin{array}{l}\text { - Marco conceptual. } \\
\text { - Audiencias y contenidos. } \\
\text { - Situación de la rendición } \\
\text { de cuentas en Ecuador y } \\
\text { América Latina. } \\
\text { - Herramientas y mecanismos. } \\
\text { - Obstáculos y desafíos. } \\
\text { - Buenas prácticas e implicancias. }\end{array}$ & Virtual & 42 \\
\hline 2011 & $\begin{array}{l}\text { Herramientas } \\
\text { para } \\
\text { transparencia } \\
\text { y rendiciones } \\
\text { de cuentas. }\end{array}$ & $\begin{array}{l}\text { - Política de transparencia. } \\
\text { - Marco legal. } \\
\text { - Herramientas para } \\
\text { el monitoreo y evaluación } \\
\text { de impacto. } \\
\text { - Modelo de gestión } \\
\text { en responsabilidad social. } \\
\text { - Comunicación para } \\
\text { la transparencia. } \\
\text { - Diálogo intersectorial. }\end{array}$ & Semipresencial & 25 \\
\hline $\begin{array}{l}2014- \\
2015\end{array}$ & \begin{tabular}{l|} 
Responsabilidad \\
social como \\
un modelo de \\
gestión que \\
fomenta y facilita \\
la transparencia \\
y rendición de \\
cuentas.
\end{tabular} & $\begin{array}{l}\text { - Modelo de gestión } \\
\text { en responsabilidad social. } \\
\text { - Reporte de sostenibilidad } \\
\text { como herramienta } \\
\text { de rendición de cuentas. } \\
\text { - Comunicación estratégica para } \\
\text { la construcción de diálogo, } \\
\text { generación de alianzas } \\
\text { y legitimidad social de las OSC. }\end{array}$ & Presencial & 23 \\
\hline 2015 & \begin{tabular}{|l|} 
Innovación y \\
transparencia en \\
organizaciones \\
de la sociedad \\
civil.
\end{tabular} & $\begin{array}{l}\text { - Transversalización } \\
\text { del enfoque de género } \\
\text { en la política pública. } \\
\text { - Participación ciudadana. } \\
\text { - Transparencia y rendición } \\
\text { de cuentas. } \\
\text { - Gestión de proyectos. }\end{array}$ & Semipresencial & 35 \\
\hline
\end{tabular}

Fuente: Appe y Barragán (2016).

Elaboración propia. 
tros universitarios, e implicó la cobertura de al menos costos logísticos y de organización académica de las universidades.

En el contexto de los casos, se pueden identificar, al menos, dos roles principales, los cuales se sistematizan en la tabla 2. El promotor, que en todos los casos fue una OSC local y los aliados, tuvieron como rol la conceptualización de los programas de capacitación, la gestión y obtención del financiamiento necesario para implementarlos, y -en dos de los casos- se encargó incluso del desarrollo de los planes de estudio e identificación de profesores. Esta situación permitió desarrollar los programas de conformidad con los planteamientos previstos por las organizaciones, fortalecer las universidades mediante la capitalización de experiencia y ofrecer al participante un programa de calidad con aval académico.

El rol de aliado fue fundamental para la operativización de los programas. Aportó el conocimiento (saberes y experiencias), respaldo y convocatoria (como el caso de las redes locales) y aval académico. Es interesante notar que las tres últimas iniciativas generaron una alianza con universidades. Por un lado, la alianza permitió contar con un aval académico, pero también beneficiarse del valor que añaden las facilidades logísticas, tecnológicas y de infraestructura. Esta relación destaca ciertamente el papel, cada vez más relevante, de la academia en la construcción y desarrollo de la sociedad civil.

Hallazgo No. 2: Uno de los objetivos más importantes que se buscaron en estos procesos fue el potencial de réplica por parte de los participantes en sus entornos cercanos, sean organizaciones beneficiarias o relacionadas, o al equipo de trabajo al interno de la propia organización, lo que se vio reflejado en las evaluaciones y medición de efectos que se realizaron en dos de ellos. Las acciones de réplica también pueden tener otra lectura: interés y necesidad existente de contar con espacios de formación y socialización de conocimientos sobre EGONG, y, de forma particular, sobre transparencia y rendición de cuentas.

Como parte de la evaluación de efectos, el primer curso analizado identificó acciones concretas que los participantes implementaron en su organización, luego de haber realizado el curso. Uno de los participantes señaló que se han hecho réplicas de lo aprendido en dos juntas parroquiales y en una asociación que está en proceso de conformación. Otro de los participantes indicó que se replicó el curso y se discutió dentro de la organización los pasos 
Tabla 2

Actores, roles y responsabilidades en los casos analizados

\begin{tabular}{|l|l|l|l|l|l|}
\hline \multicolumn{1}{|c|}{ Curso } & \multicolumn{5}{c|}{ Roles } \\
\cline { 2 - 6 } & $\begin{array}{c}\text { ONG } \\
\text { local }\end{array}$ & $\begin{array}{c}\text { ONG } \\
\text { internacional }\end{array}$ & $\begin{array}{c}\text { Universidad } \\
\text { local }\end{array}$ & $\begin{array}{c}\text { Universidad } \\
\text { internacional }\end{array}$ & $\begin{array}{c}\text { Redes } \\
\text { locales }\end{array}$ \\
\hline $\begin{array}{l}\text { Conceptos y mecanismos } \\
\text { para la transparencia y } \\
\text { rendición de cuentas. }\end{array}$ & Promotor & Aliado & & & \\
\hline $\begin{array}{l}\text { Herramientas para } \\
\text { transparencia y } \\
\text { rendiciones de cuentas. }\end{array}$ & Promotor & & Aliado & & \\
\hline $\begin{array}{l}\text { Responsabilidad social } \\
\text { como un modelo } \\
\text { de gestión que fomenta } \\
\text { y facilita la transparencia y } \\
\text { rendición de cuentas. }\end{array}$ & Promotor & & Aliado & & Aliados (2) \\
\hline $\begin{array}{l}\text { Innovación y transparencia } \\
\text { en organizaciones } \\
\text { de la sociedad civil. }\end{array}$ & Promotor & & Aliado & Aliado & \\
\hline
\end{tabular}

Fuente: Appe y Barragán (2016)

Elaboración propia.

necesarios para la elaboración de un manual de transparencia institucional (CEDA 2011).

El último caso analizado demuestra el interés de las organizaciones de seguir construyendo capacidades internas para mejorar y potenciar su gestión y el impacto de su rol social, lo que propició una segunda convocatoria para el curso.

Hallazgo No. 3: En tres de los casos, la evaluación de los programas fue un factor crítico para plantear nuevos procesos en el futuro. En un primer momento, se abordaron aspectos más generales sobre la transparencia y rendición de cuentas, brindando al participante un panorama amplio de los aspectos teóricos o ejemplos de buenas prácticas. Posteriormente, se enfocaron en herramientas más concretas y prácticas que permitan a los gestores de las 
organizaciones implementar acciones prácticas de sus organizaciones, aun cuando sean acciones de mediano y largo plazo y se vinculen con procesos más amplios como el modelo de gestión, la comunicación, la equidad de género o medición del impacto.

En el último de los casos, la evaluación permitió replantear una nueva edición del programa que incluye ajustes en contenidos y metodología. Como en todo proceso de desarrollo, los aprendizajes que se van capitalizando permiten, fortalecer los procesos y el impacto que pueden generar.

\section{Conclusiones}

Si bien los cursos ofertados son una respuesta a demandas provenientes desde las agendas de las organizaciones promotoras, las evaluaciones de los cursos reflejaron la utilidad de este tipo de procesos de EGONG así como la necesidad de contar con una oferta de formación sostenida, y que no responda a iniciativas puntuales.

Queda aún por investigar, cualitativa y cuantitativamente varios aspectos sobre la demanda de formación que existe en el país y en la región. Se debería indagar sobre las razones por las cuales las universidades no aprovechan la demanda existente, el potencial de construcción de alianzas con OSC para promover programas de EGONG, generales y especializados, inician procesos para fortalecer las mallas curriculares o dinamizan procesos de investigación y vinculación social que han sido dispuestos por la Ley Orgánica de Educación Superior.

\section{Referencias}

Appe, Susan. 2015. "Is NGO Education Matching Up to the Demand? The Case of Ecuador". Journal of Nonprofit Education and Leadership 5, No. 4: 244-260.

Appe, Susan, y Daniel Barragán. 2013. "Strategies Outside the Formal Classroom: Nonprofit Management Education in Transparency and Accountability". Journal of Public Affairs Education 19, No. 4: 591-614.

---. 2016. "Civil Society Vocabularies and Signaling Value: Cases From Colombia and Ecuador". Administrative Theory \& Praxis 39, No. 2: 100-121. 
Arcos, Carlos, y Edison Palomeque. 1997. El mito al debate: las ONGs en Ecuador, Quito: Abya-Yala.

Arias, Alicia. 2011. Situación de la transparencia y rendición de cuentas en las organizaciones no gubernamentales $(O N G)$ del Ecuador. Quito: Centro Ecuatoriano de Derecho Ambiental.

Barragán, Daniel. 2010. Rendición de cuentas: de la transparencia a la legitimidad social. Quito: Centro Ecuatoriano de Derecho Ambiental.

---. 2011. Informe de sistematización del proyecto "Hacia una gobernabilidad transparente: Fortaleciendo la transparencia y rendición de cuentas de las organizaciones de la sociedad civil”. Quito: Centro Ecuatoriano de Derecho Ambiental.

Belletini, Orazio. 2015. "Sociedad civil y bienes públicos”. En Bernardo Sorj y otros, Estado y organizaciones ciudadanas: reflexiones y experiencias para un debate necesario, 59-89. Quito: Grupo Faro.

Bies, Angela. 2010. "Evolution of Nonprofit Self-Regulation in Europe". Nonprofit and Voluntary Sector Quarterly 39, No. 6: 1057-1086.

Bies, Angela, y Amy Blackwood. 2007. “Accountability, Ethics, Evaluation, and Governance in Nonprofit Management Education: Trends and Treatment". Journal of Public Affairs Education 13, No. 3/4: 519-547.

Bonbright, David. 2007. El rostro cambiante de la rendición de cuentas de las ONG. Montevideo: Instituto de Comunicación y Desarrollo.

Bothwell, Robert. 2001. "Trends in Self-Regulation and Transparency of Nonprofits in the U.S.”. The International Journal of Not-for-Profit Law 2, No. 3: 100-115.

Brautigam, Deborah, y Monique Segarra. 2007. "Difficult Partnerships: The World Bank, States, and NGOs". Latin American Politics and Society 49, No. 4: 149-181.

Burlingame, Dwight. 2009. "Nonprofit and Philanthropic Studies Education: The Need to Emphasize Leadership and Liberal Arts”. Journal of Public Affairs Education 15, No. 1: 59-67.

Carreras, Ignasi, y Maria Sureda. 2011. Evolución de la "profesionalización” en las ONG: el impacto de los programas formativos. Barcelona: ESADE.

Centro Ecuatoriano de Derecho Ambiental (CEDA). 2011. Informe de Evaluación del curso a distancia "Un paso adelante: conceptos y mecanismos para la transparencia y la rendición de cuentas de las organizaciones de la sociedad civil”. Quito: CEDA.

---. s. f. Evaluación taller de capacitación a capacitadores sobre Ley Orgánica de Transparencia y Acceso a la Información Pública (LOTAIP). Quito: CEDA.

Chiriboga, Manuel. 2014. Las ONG ecuatorianas en los procesos de cambio. Quito: Abya-Yala / Comité Ecuménico de Proyectos.

Cruz, Anabel, e Inés Pousadela, edit. 2008. Ver a través: poder, rendición de cuentas y sociedad civil. Montevideo: Instituto de Comunicación y Desarrollo.

De Vita, Carole, Cory Fleming y Eric Twombly. 2012. "Building Nonprofit Capacity. A Framework for Addresing the Problem". En Steven Ott y Lisa Dicke, edit., Understanding 


\section{Susan Appe y Daniel Barragán}

Nonprofit Organizations. Governance, Leadership, and Management, 5-30. Philadelphia: Westview Press Books.

Donmoyer, Robert, Pat Libby, Mary McDonald y Laura Deitrick. 2012. "Bridging the Theory-practice Gap in a Nonprofit and Philanthropic Studies Master's Degree Program". Nonprofit Management and Leadership, No. 23: 93-104.

Ebrahim, Alnoor. 2003. "Accountability in Practice: Mechanisms for NGOs". World Development 31, No. 5: 813-829.

Ecuador. Ley Orgánica de Educación Superior, en Registro Oficial, Suplemento, No. 298 (Quito, de 12 de octubre de 2010).

Gugerty, Mary Kay. 2008. "The effectiveness of NGO Self-regulation: Theory and evidence from Africa". Public Administration and Development 28, No. 2: 105-118.

Heinrich, V. Finn, edit. 2007. CIVICUS Global Survey of the State of Civil Society: Volume 1. Colorado: Kumarian Press.

Indij, Damián, y Mariana Lomé. 2013. Informe necesidades de fortalecimiento institucional $y$ habilidades de gestión requeridas en las organizaciones sociales ambientales. Buenos Aires: Centro de Innovación Social de la Universidad de San Andrés.

Jackson, Terence. 2009. "A Critical Cross-cultural Perspective for Developing Nonprofit International Management Capacity". Nonprofit Management and Leadership, No. 19: 443-466.

Mirabella, Roseanne. 2007. "University-Based Educational Programs in Nonprofit Management and Philanthropic Studies: A 10-Year Review and Projections of Future Trends". Nonprofit and Voluntary Sector Quarterly 36, No. 4: 112-127.

Mirabella, Roseanne, Giuliana Gemelli, Margy-Jean Malcolm y Gabriel Berger. 2007. "Nonprofit and Philanthropic Studies: International Overview of the Field in Africa, Canada, Latin America, Asia, the Pacific, and Europe". Nonprofit and Voluntary Sector Quarterly 36, No. 4: 110-135.

Mirabella, Roseanne, y Dennis Young. 2012. "The Development of Education for Social Entrepreneurship and Nonprofit Management: Diverging or Converging paths?”. Nonprofit Management and Leadership, No. 23: 43-57.

O'Neill, Michael. 2005. "Developmental Contexts of Nonprofit Management Education". Nonprofit Management and Leadership, 16: 5-17.

Ott, Steve, y Lisa Dicke. 2012. Understanding Nonprofit Organizations. Governance, Leadership, and Management. Filadelfia: Boulder.

Prakash, Aseem, y Mary Kay Gugerty. 2010. "Trust but Verify? Voluntary Regulation Programs in the Nonprofit Sector". Regulation \& Governance 4, No. 1: 22-47.

Rubaii, Nadia, y Cristian Pliscoff. 2013. "Public Administration Education in Latin America-Understanding Teaching in Context: An Introduction to the Symposium". Journal of Public Affairs Education 19, No. 4: 585-589.

Sanabria, Pablo, Nadia Rubaii y Gabriel Purón. 2016. "Public Affairs Graduate Education in Latin America: Emulation or Identity?". Policy and Society 35, No. 4: 315-331. 
Schmitz, Hans Peter, Paloma Raggo y Tosca Bruno-van Vijfeijken. 2011. "Accountability of Transnational NGOs: Aspirations vs. Practice”. Nonprofit and Voluntary Sector Quarterly 41, No. 6: 1175-1194.

Sidel, Mark. 2005. "The Guardians Guarding Themselves: A Comparative Perspective on Self Regulation. Chicago-Kent Law Review 80, No. 2: 803-835.

Wing, Kennard. 2004. "Assessing the Effectiveness of Capacity-building Initiatives: Seven Issues for the Field”. Nonprofit and Voluntary Sector Quarterly 33, No. 1: 153-160.

Wish, Naomi. B., y Roseanne Mirabella. 1998. "Curricular Variations in Nonprofit Management Graduate Programs". Nonprofit Management and Leadership, No. 9: 99-110.

World Bank. 2005. Issues and Options for Improving Engagement Between the World Bank and Civil Society Organizations. Washington DC: The World Bank-Civil Society Team.

Young, Dennis, y Mary Clark Grinsfelder. 2011. "Social Entrepreneurship and the Financing of Third Sector Organizations". Journal of Public Affairs Education 17, No. 4: 543-567.

Zadek, Simon. 2006. Reflections on Accountability. Londres: AccountAbility.

\section{Entrevista (2016)}

Lincango, Cecilia. Coordinadora de capacidades, Grupo Faro. 